Anita R. Modi, MD

Department of Infectious Disease,

Cleveland Clinic
Christopher S. Kovacs, MD

Department of Infectious Disease, Cleveland Clinic

Assistant Professor, Cleveland Clinic Lerner College

of Medicine of Case Western Reserve University,

Cleveland, $\mathrm{OH}$

\title{
Community-acquired pneumonia: Strategies for triage and treatment
}

\section{ABSTRACT}

Community-acquired pneumonia significantly contributes to patient morbidity and healthcare costs. As our understanding of this common infection grows, collaborative efforts among researchers and clinical societies provide new literature and updated guidelines informing its management. This review discusses diagnostic methods, empiric treatment, and infection prevention strategies for patients with suspected community-acquired pneumonia.

\section{KEY POINTS}

Systematically stratifying patients with suspected community-acquired pneumonia based on mortality risk can aid in designating the safest level of care for each patient.

Empiric treatment should be informed by the local antibiogram (ie, local patterns of antibiotic resistance) with multidrug-resistant organism coverage added based on individual patient and institutional risk factors.

Prompt de-escalation to targeted antimicrobial therapy, guided by diagnostic testing, can reduce antibiotic resistance and antibiotic-related adverse drug reactions.

Appropriate clinical and radiographic follow-up after antibiotic course completion to assess for treatment failure is a subject of ongoing debate.
W HILE PHYSICIANS HAVE TREATED pneumonia for centuries, each stage of the clinical decision-making process still poses challenges, from determining the most appropriate setting of care for a patient with suspected pneumonia to planning follow-up after antibiotic completion. Over the years, physicians have witnessed the advent of new medical and respiratory therapies as well as the development of antibiotic resistance in the management of this common infection.

Inpatients with pneumonia fall into 2 categories: those with community-acquired pneumonia (CAP) who are admitted, and those who develop either hospital-acquired or ventilator-associated pneumonia while already hospitalized. Each patient population faces unique organism exposures, and thus, recommended diagnostic tests, empiric treatment regimens, and goals for infection prevention vary.

This article reviews guidelines by the Infectious Diseases Society of America (IDSA) and the American Thoracic Society (ATS) ${ }^{1}$ and interprets recent studies to address questions that arise specifically in the inpatient management of CAP.

\section{COMMON AND COSTLY}

CAP is a significant health concern, with one study reporting 915,500 episodes in adults at least 65 years of age in the United States every year, and medical costs associated with CAP exceeding $\$ 10$ billion in 2011 . $^{2,3}$

The National Center for Health Statistics reported 1.7 million visits to emergency departments in the United States in 2017 in which pneumonia was the primary discharge diagnosis, and listed pneumonia as the cause of death for 49,157 people in $2017 .{ }^{4}$ 
TABLE 1

\section{The CURB-65 calculator}

\begin{tabular}{lll} 
& Criteria & Points \\
\hline C & Confusion & 1 \\
$\mathbf{U}$ & Urea $>7 \mathrm{mmol} / \mathrm{L}$ & 1 \\
$\mathbf{R}$ & Respiratory rate $>30$ breaths per minute & 1 \\
B & $\begin{array}{l}\text { Systolic blood pressure }<90 \mathrm{~mm} \mathrm{Hg} \\
\text { or diastolic blood pressure }<60 \mathrm{~mm} \mathrm{Hg}\end{array}$ & 1 \\
$\mathbf{6 5}$ & Age $\geq 65$ & 1 \\
& & \\
& Level of care required & Total score \\
\hline & Outpatient & $0-1$ \\
& Inpatient & 2
\end{tabular}

\section{What level of care does the patient need: Outpatient? Inpatient? Intensive?}

\section{RISK-STRATIFICATION OF COMMUNITY-ACQUIRED PNEUMONIA}

The IDSA/ATS 2019 guidelines $^{1}$ emphasize the importance of first determining what level of patient care is needed: Is outpatient treatment appropriate, or does the patient need to be admitted to the hospital, or even to the intensive care unit? Appropriate triage can prevent stresses on the patient and the healthcare system associated with under- or overestimating illness severity. Patients at high risk of death whose acuity is not fully appreciated face inadequate support, while those admitted despite low risk of death may be unnecessarily subjected to the risks of the hospital setting, such as infections from healthcare-associated multidrug-resistant organisms.

Risk calculators are routinely used to help physicians triage their patients in everyday practice, although they have not been specifically validated to predict the need for admission.

CURB-65 is a simple calculator based on 5 risk factors first identified in 1987 (Table 1). ${ }^{1}$ Patients receive 1 point each for confusion, high blood urea nitrogen, high respiratory rate, low blood pressure, and age 65 or older; the higher the total score, the higher the 30-day mortality risk. According to the
IDSA/ATS, patients with scores of 0 or 1 can be managed as outpatients, those with scores of 2 should be admitted to the hospital, and those with scores of 3,4 , or 5 need care in the intensive care unit.

An abbreviated version of this calculator, CRB-65, allows risk-stratification of outpatients without laboratory work. ${ }^{1}$

The Pneumonia Severity Index incorporates 20 risk factors to place patients into 5 classes correlated with mortality risk (Table 2). ${ }^{5}$ The authors suggest outpatient management for those in classes I or II and inpatient management for those in risk classes IV and V. Patients in class III may be safely treated in an outpatient setting with adequate support or in an inpatient observation unit.

While CURB-65 may be better in busy clinical settings, as it is a shorter risk stratification scale for CAP, the Pneumonia Severity Index is preferred by the IDSA/ATS 2019 guidelines as it has been more extensively studied and validated. ${ }^{1}$

The IDSA/ATS guidelines list a separate set of major and minor criteria to define "severe pneumonia" to determine which patients with suspected CAP merit intensive care. ${ }^{1}$ At least 1 of the major criteria or at least 3 of the minor criteria are required for the diagnosis of severe pneumonia (Table 3 ).

The Pneumonia Patient Outcomes Research Team study, a multicenter, prospective controlled study of both ambulatory and hospitalized patients with CAP, also devised a list of risk factors associated with death within 30 days. ${ }^{6}$ These risk factors include altered mental status, uremia, leukopenia, and hypoxemia. Chronic liver failure was a risk factor highlighted in this study but was not included in the IDSA/ATS criteria.

Yet none of these scoring systems can fully capture all medical or psychosocial comorbidities that may prevent successful recovery in the outpatient setting. A retrospective chart review of more than 1,800 patients found that $45 \%$ of patients who had "low-risk" CAP by the Pneumonia Severity Index were nevertheless admitted. ${ }^{7}$ Patients with cognitive impairment, coronary artery disease, diabetes mellitus, pulmonary disease, multilobular radiographic opacities, home oxygen therapy, corticosteroid use, or use of antibiotics prior 
to presentation had increased odds of hospitalization.

Clinical judgment should be applied to the results of any of these calculators to appropriately triage patients with pneumonia.

\section{DIAGNOSIS}

OF COMMUNITY-ACQUIRED PNEUMONIA

\section{Imaging}

After triaging a patient with suspected CAP to the safest level of care, several radiographic and laboratory methods can be used to verify the diagnosis and identify the organism most likely responsible for the ongoing infection. Chest radiographs with demonstrable infiltrates are required to diagnose CAP and to distinguish it from upper respiratory tract infection. ${ }^{1}$

Different organisms can be associated with characteristic infiltrate patterns, which often manifest within 12 hours of symptom onset:

Focal nonsegmental or lobar pneumonia (Figure 1). Typical bacterial pneumonias caused by organisms such as Streptococcus pneumoniae tend to manifest with an airspace opacity in 1 segment or lobe, though antibiotic use can alter their pathophysiology to create a patchy, multilobular opacity pattern.

Multifocal bronchopneumonia or lobar pneumonia. Bronchopneumonias, similarly characterized by a patchy pattern, are most commonly caused by Staphylococcus aureus, Haemophilus influenzae, and fungi. ${ }^{8}$

Focal or diffuse "interstitial" pneumonia (Figure 2). Atypical bacterial organisms including Legionella pneumophila, Mycoplasma pneumoniae, and Chlamydophila pneumoniae frequently involve the lung bases in a diffuse, bilateral, reticulonodular pattern, but can start as isolated lobar opacities on chest radiography. ${ }^{9}$ Viral organisms are associated with diffuse, bilateral lung involvement as well.

Early radiographic identification of pulmonary complications, such as pleural effusions or cavitating lesions, can provide more clues to the causative organism and allow for timely intervention. ${ }^{9}$

\section{How accurate is chest radiography?}

The utility of chest radiographs in diagnosing CAP is ultimately subject to interobserver variability, with some studies citing $65 \%$ ac-
TABLE 2

\section{Pneumonia Severity Index calculator and associated risk classes}

Risk factor

Points

\section{Demographics}

Men

Age (years)

Women

Nursing home resident

Age (years) -10

$+10$

\section{Comorbidities}

Neoplasm

$+30$

Liver disease

$+20$

Heart failure $\quad+10$

Stroke +10

Renal failure

$+10$

\section{Physical examination findings}

Altered mental status $\quad+20$

Respiratory rate $>30$ breaths per minute $\quad+20$

Systolic blood pressure $<90 \mathrm{~mm} \mathrm{Hg} \quad+20$

Temperature $<95^{\circ} \mathrm{F}$ or $>104^{\circ} \mathrm{F}+15$

Heart rate $>125$ beats per minute $\quad+10$

\section{Laboratory and radiographic findings}

Arterial $\mathrm{pH}<7.35+30$

Blood urea nitrogen $>30 \mathrm{mg} / \mathrm{dL} \quad+20$

Sodium $<130 \mathrm{mmol} / \mathrm{L} \quad+20$

Glucose $>250 \mathrm{mg} / \mathrm{dL} \quad+10$

Hematocrit $<30 \% \quad+10$

Partial pressure of arterial oxygen $<60 \mathrm{~mm} \mathrm{Hg} \quad+10$

Pleural effusion $\quad+10$

\begin{tabular}{ll} 
Risk class & Total points \\
\hline I & $<51$ \\
II & $51-70$ \\
III & $71-90$ \\
IV & $91-130$ \\
V & $>130$
\end{tabular}

From Fine MJ, Auble TE, Yealy DM, et al. A prediction rule to identify low-risk patients with community-acquired pneumonia. N Engl J Med 1997; 336(4):243-250. Copyright 1997, Massachusetts Medical Society. Reprinted with permission from the Massachusetts Medical Society.

curacy in diagnosing viral pneumonia, $67 \%$ in diagnosing bacterial pneumonia, and no statistical reliability for differentiating bacterial 
TABLE 3

\section{Severe pneumonia: Infectious Diseases Society of America and American Thoracic Society criteria}

\section{Major criteria}

Respiratory distress requiring mechanical ventilation

Septic shock

\section{Minor criteria}

Confusion

Respiratory rate $>30$ breaths per minute

Blood urea nitrogen $>7 \mathrm{mmol} / \mathrm{L}$

Leukopenia resulting from infection

Thrombocytopenia

Hypothermia

Hypotension requiring aggressive fluids

$\mathrm{PaO}_{2} / \mathrm{FiO}_{2}<250$

Multilobar infiltrates

Having at least 1 major criterion or at least 3 minor criteria suggests the need for intensive care.

From Mandell LA, Wunderink RG, Anzueto A, et al; Infectious Diseases Society of America; American Thoracic Society. Infectious Diseases Society of America/American Thoracic Society consensus guidelines on the management of community-acquired pneumonia in adults. Clin Infect Dis 2007: 44(suppl 2):S27-S72, by permission of Oxford University Press.

from nonbacterial pneumonias. ${ }^{10}$ A Swedish retrospective chart review of 103 outpatients with suspected CAP noted that just $88 \%$ of patients with high clinical concern for CAP demonstrated radiographic evidence of infection. ${ }^{11}$

\section{Microbiology}

A thorough social history should be gathered for every patient with suspected CAP to screen for potential occupational, travel, or endemic exposures. This will guide microbiologic testing and empiric antibiotic treatment. ${ }^{1}$ For example, patients presenting during flu season or with known exposures to poultry in areas of prior influenza outbreaks should be screened for influenza $A$ and $B$ with a nasopharyngeal swab.

Isolating a specific organism in outpatients with CAP may not be necessary but is recommended to guide de-escalation of empiric an-

\section{TABLE 4}

\section{Indications for blood culture testing in suspected community-acquired pneumonia}

Intensive care unit admission

Cavitary infiltrates

Leukopenia

Active alcohol abuse

Chronic liver failure

Asplenia (anatomic or functional)

Positive pneumococcal urine antigen test

Pleural effusion

Based on information in reference 1

tibiotic regimens. ${ }^{1}$ Pretreatment Gram stain and culture in patients able to adequately expectorate a good-quality specimen or endotracheal aspirate in intubated patients should be collected. Patients fulfilling criteria for severe pneumonia as defined by the IDSA/ATS guidelines merit blood and sputum cultures as well as urinary antigen tests for $L$ pneumophila and S pneumoniae (Table 4). ${ }^{1}$

Overall, active surveillance of more than 2,200 patients with CAP requiring hospitalization noted that $38 \%$ of blood and sputum cultures, nasopharyngeal and oropharyngeal swabs, and urinary antigens yielded a causative organism. ${ }^{12}$ Viral organisms accounted for $25 \%$ of these cases and bacterial organisms accounted for $14 \% ; 5 \%$ of patients with viral pneumonias were coinfected with either another respiratory virus or a bacterial organism.

\section{Procalcitonin testing}

Procalcitonin testing can help differentiate viral from bacterial pathogens in patients admitted for CAP, preventing the use of unnecessary antibiotics and allowing prompt de-escalation of empiric therapy more effectively than clinical judgment alone. ${ }^{13}$ While any infectious pneumonia can precipitate elevations of this serum biomarker, typical bacteria tend to result in higher procalcitonin levels than atypical bacteria or viruses. ${ }^{14}$ Cytokines, associated with bacterial infections, enhance procalcitonin release, while interferons, associated with 
viral infections, inhibit procalcitonin release. This biomarker is not perfect, however, and will not be elevated in up to $23 \%$ of typical bacterial infections. ${ }^{14}$

For this reason, procalcitonin should not replace clinical judgment in guiding the decision to initiate antimicrobial therapy for patients with suspected CAP but can be used in conjunction with clinical judgment to de-escalate therapy. In patients whose clinical histories suggest alternative causes of respiratory distress or improvement with concomitantly administered therapies such as diuresis, a negative procalcitonin can help guide cessation of antibiotics. On the other hand, in patients with polymerase chain reaction-proven influenza, an elevated procalcitonin can suggest continuation of antibiotics to treat bacterial superinfection.

\section{MANAGEMENT}

OF COMMUNITY-ACQUIRED PNEUMONIA

\section{Antibiotic therapy}

The selection of antibiotics before a causative pathogen is identified should be informed by the patient's risk factors and degree of illness (Table 5, Table 6). ${ }^{1}$

Patients on a medical floor should be started on either a respiratory fluoroquinolone or a combination of a beta-lactam plus a macrolide; intensive care patients should receive a beta-lactam plus either a macrolide or a respiratory fluoroquinolone. Doxycycline can be used as an alternative to the macrolide or respiratory fluoroquinolone to cover atypical organisms such as Chlamydia pneumoniae, Legionella pneumophila, and Mycoplasma pneumoniae in patients with prolonged QTc. In penicillin-allergic patients, aztreonam should be used in combination with an aminoglycoside and a respiratory fluoroquinolone.

Patients who may have been exposed to influenza or who have a history of injection drug use or structural lung disease or who have a lung abscess, cavitary infiltrates, or endobronchial obstruction also merit coverage against community-acquired methicillin-resistant $S$ aureus (MRSA) with vancomycin or linezolid. Those with confirmed or suspected influenza A presenting within 48 hours of symptom

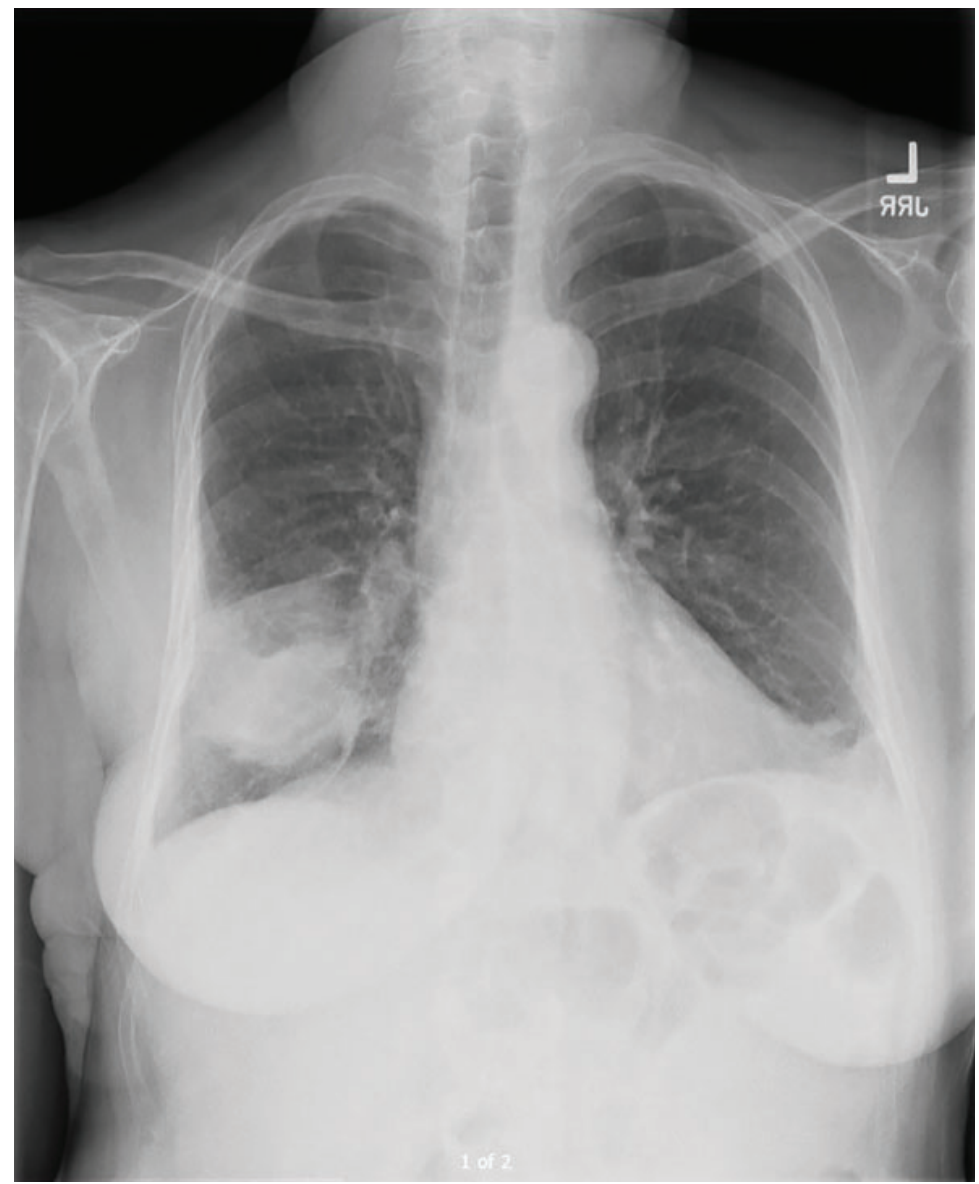

Figure 1. Focal lobar pneumonia.

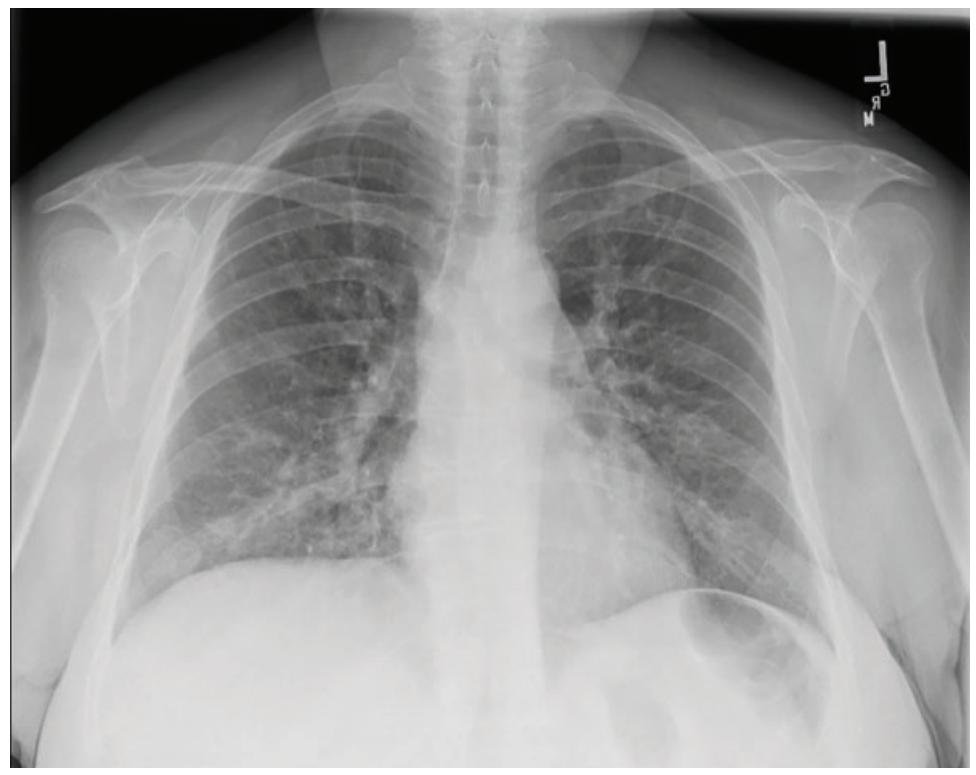

Fgure 2. Diffuse interstitial pneumonia. 


\section{TABLE 5}

\section{Common organisms in community-acquired pneumonia}

\section{Outpatient care}

Streptococcus pneumoniae

Mycoplasma pneumoniae

Haemophilus influenzae

Chlamydophila pneumoniae

Respiratory virus (influenza $A$ and $B$, adenovirus, respiratory syncytial virus, parainfluenza)

Inpatient (non-intensive care)

$S$ pneumoniae

M pneumoniae

C pneumoniae

$H$ influenzae

Legionella species

Aspiration-related oral flora

Respiratory viruses

\section{Inpatient (intensive care)}

Use clinical

judgment

when triaging

patients with

community-

acquired

pneumonia
$S$ pneumoniae

Staphylococcus aureus

Legionella species

Gram-negative bacilli

\section{$H$ influenzae}

From Mandell LA, Wunderink RG, Anzueto A, et al; Infectious Diseases Society of America; American Thoracic Society Infectious Diseases Society of America/American Thoracic Society consensus guidelines on the ety of America/American Thoracic Society consensus guidelines on the
management of community-acquired pneumonia in adults. Clin Infect Dis 2007; 44(suppl 2):S27-S72, by permission of Oxford University Press.

onset or with severe illness should be treated with oseltamivir. ${ }^{1}$

If an organism is identified by culture, polymerase chain reaction, or serology, the empiric antibiotic regimen should be tailored to this organism. MRSA nares screening can be reliably used to guide empiric and targeted antimicrobial regimens; patients started on vancomycin or linezolid based on the abovestated risk factors can be safely de-escalated on the basis of a negative nasal swab. ${ }^{15}$ The pneumococcal urinary antigen has a similarly
TABLE 6

\section{Initial antibiotic therapy for community-acquired pneumonia}

\author{
Outpatients without comorbidities ${ }^{a}$ \\ Amoxicillin \\ Or doxycycline \\ Or a macrolide
}

\section{Outpatients with comorbidities \\ Combination therapy: \\ Amoxicillin/clavulanate or a cephalosporin \\ Plus a macrolide or doxycycline \\ Or monotherapy with a fluoroquinolone}

\section{Patients on a medical floor}

A fluoroquinolone

Or a combination of a beta-lactam plus a macrolide

\section{Intensive care patients}

A beta-lactam

Plus either a macrolide or a fluoroquinolone

\section{Add coverage as needed for:}

Methicillin-resistant Staphylococcus aureus (MRSA)

Pseudomonas aeruginosa

Influenza A

aComorbidities include heart, lung, liver, or renal disease,

diabetes mellitus, alcoholism, malignancy, and asplenia

Based on information from reference 1 .

reliable negative predictive value and can also be used to de-escalate empiric antimicrobial therapy. ${ }^{16}$

Should microbiologic evaluation fail to identify a causative organism, the patient's individual risk factors as listed above must be considered in de-escalating therapy to a final regimen with coverage for MRSA, Pseudomonas aeruginosa, or atypical pathogens as indicated. Pseudomonal pneumonia has been associated with higher risk of mortality and relapse than pneumonia caused by other pathogens.

\section{Corticosteroids as adjunctive therapy}

The use of adjunctive corticosteroids for CAP management has been widely contested. The IDSA/ATS guidelines recommend against corticosteroid use for adjunctive treatment of CAP except in patients with refractory septic shock. ${ }^{1}$

\section{Later management}

Patients who are hemodynamically stable, can ingest medications safely, and have a normal gastrointestinal tract can be discharged 
on oral therapy without waiting to observe the clinical response. Antibiotics should be given for at least 5 days, though longer durations may be needed in immunocompromised patients or in those with pulmonary or extrapulmonary complications. ${ }^{1}$

An infectious disease consultation may be beneficial if long-term intravenous antibiotic therapy is anticipated or if the patient progressively deteriorates on guideline-based antimicrobial therapy.

Pulmonary consultation may be needed for bronchoscopy to obtain deep respiratory samples, especially if the patient is clinically worsening and the causative pathogen remains unidentified. We acknowledge that the yield of bronchoscopy and bronchoalveolar lavage samples is reduced with longer durations of antibiotic therapy, yet believe that in the context of clinical worsening in spite of antibiotics, bronchoalveolar lavage may help successfully identify multidrug-resistant or

\section{REFERENCES}

1. Metlay JP, Waterer GW, Long AC, et al. Diagnosis and treatment of adults with community-acquired pneumonia. An official clinical practice guideline of the American Thoracic Society and Infectious Diseases Society of America. Am J Respir Crit Care Med 2019; 200(7):e45e67. doi:10.1164/rccm.201908-1581ST

2. Jackson ML, Neuzil KM, Thompson WW, et al. The burden of community-acquired pneumonia in seniors: results of a population-based study. Clin Infect Dis 2004; 39(11):1642-1650. doi:10.1086/425615

3. Pfuntner A, Wier LM, Steiner C. Costs for hospital stays in the United States, 2011. HCUP Statistical Brief \#168. December 2013. Agency for Healthcare Research and Quality, Rockville, MD. www.hcup-us.ahrq.gov/reports/ statbriefs/sb168-Hospital-Costs-United-States-2011.pdf. Accessed February 6, 2020.

4. Centers for Disease Control and Prevention (CDC). National Center for Health Statistics. Pneumonia. cdc.gov/ nchs/fastats/pneumonia.htm. Accessed February 6, 2020.

5. Fine MJ, Auble TE, Yealy DM, et al. A prediction rule to identify low-risk patients with community-acquired pneumonia. N Engl J Med 1997; 336(4):243-250. doi:10.1056/NEJM199701233360402

6. Mortensen EM, Coley CM, Singer DE, et al. Causes of death for patients with community-acquired pneumonia: results from the Pneumonia Patient Outcomes Research Team cohort study. Arch Intern Med 2002; 162(9):1059-1064. doi:10.1001/archinte.162.9.1059

7. Labarere J, Stone RA, Scott Obrosky D, et al. Factors associated with the hospitalization of low-risk patients with community-acquired pneumonia in a clusterrandomized trial. J Gen Intern Med 2006; 21(7):745-752. doi:10.1111/j.1525-1497.2006.00510.x

8. Franqut $\mathbf{T}$. Imaging of community-acquired pneumonia. J Thorac Imaging 2018; 33(5):282-294. doi:10.1097/RTI.0000000000000347 atypical pathogens which may not be covered by the ongoing antibiotic regimen. Pulmonology consultation is also indicated for patients with complications of pneumonia such as empyema that require procedural intervention.

\section{TAKE-HOME POINTS}

- CAP continues to contribute to patient morbidity and mortality as well as healthcare costs.

- Professional societies have released collaborative guidelines to streamline practice patterns and provide evidence-based protocols for the diagnosis, treatment, and prevention of this common infection.

- Further research is needed to delineate appropriate strategies to de-escalate antibiotics in the absence of a causative organism, define the dose and duration of adjunctive steroid use, and clarify patient follow-up after discharge from the hospital.

9. Vilar J, Domingo ML, Soto C, Cogollos J. Radiology of bacterial pneumonia. Eur J Radiol 2004; 51(2):102-113. doi:10.1016/j.ejrad.2004.03.010

10. Katz DS, Leung AN. Radiology of pneumonia. Clin Chest Med 1999; 20(3):549-562. pmid:10516903

11. Moberg AB, Taléus U, Garvin P, Fransson SG, Falk M. Community-acquired pneumonia in primary care: clinical assessment and the usability of chest radiography. Scand J Prim Health Care 2016; 34(1):21-27. doi:10.3109/02813432.2015.1132889

12. Jain S, Self WH, Wunderink RG, et al; CDC EPIC Study Team. Community-acquired pneumonia requiring hospitalization among US adults. N Engl J Med 2015; 373(5):415-427. doi:10.1056/NEJMoa1500245

13. Schuetz P, Christ-Crain M, Thomann R, et al; ProHOSP Study Group. Effect of procalcitonin-based guidelines vs standard guidelines on antibiotic use in lower respiratory tract infections: the ProHOSP randomized controlled trial. JAMA 2009; 302(10):1059-1066. doi:10.1001/jama.2009.1297

14. Self WH, Balk RA, Grijalva CG, et al. Procalcitonin as a marker of etiology in adults hospitalized with community-acquired pneumonia. Clin Infect Dis 2017; 65(2):183190. doi:10.1093/cid/cix317

15. Parente DM, Cunha CB, Mylonakis E, Timbrook TT. The clinical utility of methicillin-resistant Staphylococcus aureus (MRSA) nasal screening to rule out MRSA pneumonia: a diagnostic meta-analysis with antimicrobial stewardship implications. Clin Infect Dis 2018; 67(1):1-7. doi:10.1093/cid/ciy024

16. West DM, McCauley LM, Sorensen JS, Jephson AR, Dean NC. Pneumococcal urinary antigen test use in diagnosis and treatment of pneumonia in seven Utah hospitals. ERJ Open Res 2016; 2(4). pii:00011-2016. doi:10.1183/23120541.00011-2016

Address: Anita R. Modi, MD, Department of Infectious Disease, G21, Cleveland Clinic, 9500 Euclid Avenue, Cleveland, $\mathrm{OH}$ 44106; modia@ccf.org 